\title{
Correction to: Investigating the Role of VR in a Simulation-Based Medical Planning System for Coronary Interventions
}

Madhurima Vardhan, Harvey Shi, John Gounley, S. James Chen, Andrew Kahn, Jane Leopold, and Amanda Randles

\section{Correction to: \\ Chapter "Investigating the Role of VR in a Simulation-Based Medical Planning System for Coronary Interventions" in: D. Shen et al. (Eds.): Medical Image Computing and Computer Assisted Intervention - MICCAI 2019, LNCS 11768, https://doi.org/10.1007/978-3-030-32254-0_41}

The original version of this chapter was revised. The spelling of the last author's name was corrected to Amanda Randles. 